\title{
Associação entre Dinamometria Manual, Estado Nutricional e Complicações Pós-Operatórias em Pacientes Oncológicos
} Association between Manual Dynamometer, Nutritional Status and Postoperative Complications in Oncologic Patients

Asociación entre Dinamometría Manual, Situación Nutricional y las Complicaciones Postoperatorias en Pacientes con Cáncer

\author{
Vanessa Regina Limberger'; Carla Alberici Pastore ${ }^{2}$; Renata Torres Abib ${ }^{3}$
}

\section{Resumo}

Introduçáo: A dinamometria manual é um método de avaliação nutricional e marcador de prognóstico em pacientes oncológicos pré-cirúrgicos. Objetivos: Avaliar a associação entre dinamometria manual, perfil sociodemográfico e clínico, e desfecho da internação de pacientes submetidos à cirurgia em um hospital público do Sul do Brasil. Método: Estudo longitudinal observacional, realizado no Hospital Escola da Universidade Federal de Pelotas, entre maio e novembro de 2013, com pacientes portadores de câncer submetidos à primeira cirurgia oncológica. O estado nutricional foi avaliado através do Índice de Massa Corporal e da Avaliação Subjetiva Global Produzida pelo Paciente, a força do aperto de mão foi aferida por meio de dinamometria manual e as complicaçôes foram identificadas em prontuário dos pacientes no decorrer da internaçáo. Resultados: Foram avaliados 23 pacientes, sendo 52,2\% do sexo masculino, com $47,8 \%$ da amostra apresentando câncer de cólon e reto. Foi encontrada associação entre a força do aperto de mão e o estado nutricional segundo Avaliação Subjetiva Global Produzida pelo Paciente conforme as categorias: bem nutrido, risco de desnutrição e desnutrição severa e pontuação obtida, assim como a Avaliação Subjetiva Global Produzida pelo Paciente associou-se com a localização tumoral. As complicaçóes pós-operatórias ocorreram em 21,7\% dos pacientes; porém sem associação estatística com a força do aperto de mão. Conclusão: A dinamometria manual associou-se com estado nutricional segundo Avaliaçáo Subjetiva Global Produzida pelo Paciente, diminuindo conforme a sua piora; porém não esteve associada a complicaçóes pós-operatórias, tempo de internação ou mortalidade nessa amostra.

Palavras-chave: Dinamômetro de Força Muscular; Força da Mão; Avaliaçấo Nutricional, Pacientes Internados; Neoplasias; Estudo Observacional

\footnotetext{
${ }^{1}$ Nutricionista pela Universidade Federal de Pelotas (UFPel). Residente do Programa de Residência Integrada Multiprofissional em Saúde - Área de concentração em Atenção à Saúde Oncológica (RIMS-UFPel). E-mail: limberger.v@gmail.com.

${ }^{2}$ Doutora em Saúde e Comportamento pela Universidade Católica de Pelotas (UCPel). Nutricionista da Faculdade de Nutrição da UFPel. E-mail: pastorecarla@yahoo.com.br.

${ }^{3}$ Doutora em Ciências Biológicas: Bioquímica pela Universidade Federal do Rio Grande do Sul (UFRGS). Professora-Adjunta da Faculdade de Nutrição da UFPel. E-mail: renata.abib@ymail.com.

Endereço para correspondência: Vanessa Regina Limberger. Avenida João Pessoa, 660 - Santo Inácio. Santa Cruz do Sul (RS), Brasil. CEP: 96820-066.

E-mail: limberger.v@gmail.com.
} 


\section{INTRODUÇÃO}

Nas últimas décadas, em virtude do grande crescimento e envelhecimento da população mundial, o câncer vem se constituindo um importante problema de saúde pública, principalmente nos países em desenvolvimento. Atualmente, o câncer é considerado a segunda causa de morte no mundo e as estimativas para o ano de 2030 indicam a ocorrência de 21,4 milhōes de novos casos ${ }^{1-2}$.

No ambiente hospitalar, a prevalência de desnutrição ainda é elevada, presente em $40 \%$ a $80 \%$ dos $\operatorname{casos}^{3-4}$, sendo um processo contínuo que se desencadeia com a ingestão inadequada de nutrientes e progride por meio de uma sequência de alterações funcionais e de composição corporal'5 Já a caquexia associada ao câncer é uma síndrome multifatorial, na qual há perda contínua de massa muscular (acompanhada ou não de perda de massa gorda), que não pode ser totalmente revertida pela terapia nutricional convencional ${ }^{6-7}$.

Sabe-se que a capacidade muscular de indivíduos desnutridos encontra-se significativamente diminuída, uma vez que a desnutrição calórico-proteica afeta diretamente a perda de todas as fibras musculares esqueléticas $^{8}$ e, consequentemente, a diminuiçâao da força muscular?.

As consequências do déficit nutricional estão estreitamente relacionadas à evolução clínica desse paciente, com maior risco de complicaçôes pós-operatórias, diminuição da qualidade de vida, aumento na morbimortalidade, no tempo de internação e nos custos ao sistema de saúde ${ }^{10}$. Cerca de $20 \%$ dos óbitos em pacientes oncológicos ocorrem devido a complicaçóes decorrentes da desnutrição e não da doença propriamente dita ${ }^{11}$; portanto, a avaliaçáo e o acompanhamento nutricional tornam-se fundamentais ${ }^{3}$.

A dinamometria manual (DM) ou força do aperto de mão (FAM) é um método utilizado na avaliação nutricional dos pacientes por ser um marcador de prognóstico, inclusive em pacientes oncológicos ${ }^{8}$. É um método de fácil aplicabilidade, simples, rápido, de baixo custo, além de prever o status funcional muscular. Dessa forma, a dinamometria como método de avaliaçáo nutricional em pacientes oncológicos pré-cirúrgicos torna-se de extrema importância, uma vez que contribui para um diagnóstico nutricional mais fidedigno, a fim de identificar o risco da desnutrição com maiores chances de complicaçóes, mortalidade no período pós-operatório e maior tempo de internação hospitalar.

Diante do exposto, este estudo teve como objetivo avaliar a associação da DM com o perfil sociodemográfico e clínico, e o desfecho da internação de pacientes oncológicos submetidos à cirurgia em um hospital público do Sul do Brasil.

\section{MÉTODO}

Trata-se de um estudo longitudinal observacional, realizado de maio a novembro de 2013 no Setor de Cirurgia do Hospital Escola da Universidade Federal de Pelotas (UFPel). Foram elegíveis os pacientes maiores de 18 anos, de ambos os sexos, com diagnóstico de câncer que se internaram para a realização da primeira cirurgia oncológica e livre de limitaçôes que comprometessem a coleta de dados. Não foram incluídas as mulheres com câncer de mama ou útero por estas terem se internado no Setor de Ginecologia para realizar a cirurgia oncológica. Os dados foram coletados após o esclarecimento sobre a pesquisa, leitura e assinatura do Termo de Consentimento Livre e Esclarecido pelos pacientes.

Os pacientes foram avaliados em até 48 horas após a admissão hospitalar, quando responderam a um questionário sobre hábitos de vida, como tabagismo, etilismo e características sociodemográficas, como sexo, idade, cor e estado civil. Para análise socioeconômica, foi utilizado o instrumento proposto pela Associaçáo Brasileira de Empresas de Pesquisas (ABEP) ${ }^{12}$, classificando o indivíduo em cinco classes, variando de maior poder aquisitivo (A) ao de menor poder (E). Informaçóes referentes à doença, como localização do tumor, tipo de tratamento, presença de complicaçôes pós-operatórias, tempo de internação e a evolução clínica do pacientes (alta ou óbito), foram obtidas do prontuário médico.

Para a obtenção do peso, foi utilizada balança Filizola ${ }^{\bullet}$ digital modelo PL 150, com capacidade de $150 \mathrm{~kg}$ e precisão de 100 g. Para a medição da altura, foi utilizado estadiômetro de metal, com $200 \mathrm{~cm}$ e precisão de $1 \mathrm{~mm}$, acoplado à balança. O Índice de Massa Corporal (IMC) foi obtido pelo quociente do peso (em quilogramas), pela estatura (em metros) ao quadrado. Para os indivíduos com idade entre 18 e 59 anos, foi utilizada a classificação da Organização Mundial da Saúde ${ }^{13}$, sendo baixo peso (IMC $\leq 18,5 \mathrm{Kg} / \mathrm{m}^{2}$ ), eutrofia (IMC $\geq 18,5$ e $<25 \mathrm{Kg} / \mathrm{m}^{2}$ ), sobrepeso (IMC $\geq 25$ e $<30 \mathrm{Kg} / \mathrm{m}^{2}$ ) e obesidade (IMC $\geq 30 \mathrm{Kg} / \mathrm{m}^{2}$ ); enquanto, para os indivíduos com idade igual ou superior a 60 anos, foi utilizada a classificação sugerida por Lipschitz ${ }^{14}$, sendo baixo peso $\left(\mathrm{IMC} \leq 22 \mathrm{Kg} / \mathrm{m}^{2}\right)$, eutrofia (IMC>22 e $\left.<27 \mathrm{Kg} / \mathrm{m}^{2}\right)$ e sobrepeso $\left(\mathrm{IMC} \geq 27 \mathrm{Kg} / \mathrm{m}^{2}\right.$ ).

$\mathrm{O}$ estado nutricional também foi avaliado por meio da Avaliação Subjetiva Global Produzida pelo Paciente (ASG-PPP), versão traduzida e validada para o Brasil por Gonzalez et $\mathrm{al}^{15}$. A partir dessa ferramenta, gera-se um escore numérico de risco nutricional, resultando em diferentes níveis indicativos de intervenção nutricional. A obtenção de maiores escores indica maior risco nutricional. $\mathrm{O}$ escore entre 0 e 1 ponto indica que não há necessidade de intervenção naquele momento; entre 2 e 3 pontos, 
deve-se abordar o paciente e seu familiar para a educação nutricional; entre e 4 e 8 , deve-se realizar uma intervenção nutricional; e igual ou acima de 9 pontos, existe uma necessidade crítica de intervençáo nutricional. $\mathrm{O}$ paciente também pode ser classificado como A (bem nutrido), B (suspeita ou desnutrição moderada) ou C (gravemente desnutrido).

A aferição da FAM foi realizada por um avaliador treinado, utilizando o dinamômetro mecânico $J A M A R^{\circ}$. Os pacientes executaram o teste em posição sentada, com o cotovelo flexionado em angulo de $90^{\circ}$, antebraço e pulso em posição neutra. Os sujeitos foram instruídos a realizar três contraçóes isométricas máximas, com uma breve pausa entre as medidas. Foram obtidas três medidas de cada mão (dominante e não dominante) e a média aritmética foi utilizada como medida final ${ }^{16}$. Os valores da medida da FAM foram agrupados como máo dominante (direita nos destros, e esquerda nos canhotos) e não dominante (esquerda nos destros, e direita nos canhotos).

Os dados coletados foram digitados em planilha do software Microsoft Excel e exportados e analisados no programa $S T A T A^{\oplus}$, versão 11.1. As variáveis foram inicialmente descritas como percentuais e frequências relativas, ou como média e desvio-padrão, dependendo da natureza da variável (categórica ou contínua, respectivamente). Os testes estatísticos utilizados foram teste $t$, ANOVA e correlação de Pearson. O nível de significância considerado foi de 5\% para todos os testes. Este estudo foi aprovado pelo Comitê de Ética em Pesquisa da Faculdade de Medicina da UFPel sob o no 265.561, e segundo o ofício no 00409/13 de 24 de abril de 2013.

\section{RESULTADOS}

A amostra foi composta por 23 pacientes com idade média de 59,1 $\pm 9,9$ anos, sendo a maioria $(52,2 \%)$ do sexo masculino. O sítio tumoral mais prevalente foi cólon e reto $(47,8 \%)$, seguido de órgãos e glândulas reprodutivas, estômago, esôfago e rim e bexiga.

Em relação à avaliação do estado nutricional segundo ASG-PPP, a maioria dos pacientes $(60,9 \%)$ estava com suspeita de desnutrição ou desnutriçáo moderada, e 13,1\% com desnutrição severa. Conforme o IMC, 47,8\% apresentaram excesso de peso. Na Tabela 1, estâo descritas as características da amostra estudada.

A média para o tempo de internação foi de $23 \pm 9,8$ dias. A prevalência de complicaçôes foi de $21,7 \%$, sendo que a causa de maior ocorrência foi deiscência de anastomose $(8,7 \%)$, seguida de fístula, peritonite, infecção de ferida operatória e sepse, com $4,3 \%$ cada. Houve somente um caso de óbito. $\mathrm{O}$ pequeno tamanho amostral deste estudo se deveu a vários fatores, como o desconhecimento
Tabela 1. Características da amostra. Pelotas, 2013

\begin{tabular}{|c|c|c|}
\hline Características & $\mathbf{N}$ & $\%$ \\
\hline \multicolumn{3}{|l|}{ Sexo } \\
\hline Masculino & 12 & 52,2 \\
\hline Feminino & 11 & 47,8 \\
\hline \multicolumn{3}{|l|}{ Cor da pele } \\
\hline Branca & 20 & 87,0 \\
\hline Não branca & 3 & 13,0 \\
\hline \multicolumn{3}{|l|}{ Situação conjugal } \\
\hline Com companheiro(a) & 16 & 69,6 \\
\hline Sem companheiro(a) & 7 & 30,4 \\
\hline \multicolumn{3}{|l|}{ Tabagismo } \\
\hline Sim & 3 & 13,1 \\
\hline Não & 13 & 56,5 \\
\hline Ex-tabagista & 7 & 30,4 \\
\hline \multicolumn{3}{|l|}{ Etilismo } \\
\hline Sim & 2 & 8,7 \\
\hline Não & 18 & 78,3 \\
\hline Ex-etilista & 3 & 13,0 \\
\hline \multicolumn{3}{|l|}{ Classe social } \\
\hline $\mathrm{C}$ & 20 & 87,0 \\
\hline D & 3 & 13,0 \\
\hline
\end{tabular}

\section{Sítio tumoral}

\begin{tabular}{l|c|c}
\hline Cólon e reto & 11 & 47,8 \\
\hline Órgãos e glândulas reprodutivas & 5 & 21,7 \\
\hline Estômago & 3 & 13,1 \\
\hline Esôfago & 2 & 8,7 \\
\hline Rim e bexiga & 2 & 8,7 \\
\hline
\end{tabular}

Índice de Massa Corporal (IMC)

\begin{tabular}{l|c|c}
\hline Baixo peso & 5 & 21,8 \\
\hline Eutrofia & 7 & 30,4 \\
\hline Sobrepeso & 4 & 17,4 \\
\hline Obesidade & 7 & 30,4 \\
\hline
\end{tabular}

Avaliação Subjetiva Global (ASG-PPP)

\begin{tabular}{l|c|c}
\hline Bem nutrido (A) & 6 & 26,1 \\
\hline Suspeita ou desnutrição moderada (B) & 14 & 60,8 \\
\hline Gravemente desnutrido (C) & 3 & 13,1 \\
\hline
\end{tabular}

\section{Escore numérico}

\begin{tabular}{l|c|c}
\hline $\begin{array}{l}0-1: \text { Não há necessidade de } \\
\text { intervenção }\end{array}$ & 3 & 13,1 \\
\hline $\begin{array}{l}\text { 2- 3: Educação do paciente e seus } \\
\text { familiares }\end{array}$ & 3 & 13,1 \\
\hline $\begin{array}{l}4-8: \text { Necessita intervenção } \\
\text { nutricional }\end{array}$ & 2 & 8,7 \\
\hline $\begin{array}{l}\text { ? 9: Necessidade crítica de } \\
\text { intervenção nutricional }\end{array}$ & 15 & 65,1 \\
\hline
\end{tabular}

* Órgãos e glândulas reprodutivas: Câncer do corpo do pênis, próstata, testículo, ovário. 
do diagnóstico antes da cirurgia, ausência de exames que comprovassem o diagnóstico (biópsia) e algumas cirurgias foram de caráter diagnóstico e/ou laparotomia exploratória.

A maior parte dos pacientes era destra (91,3\%). A força do aperto da mão dominante atingiu média de 24,8 13 ,2Kgf, sendo o mínimo de 19,0Kgf e o máximo de 30,5Kgf; e da não dominante, $22,8 \pm 12,1 \mathrm{Kgf}$, sendo o mínimo de 17,6 Kgf e o máximo de 28,0 Kgf ( $\mathrm{p}=0,001$ ). A FAM da mão dominante variou significativamente entre os gêneros $(\mathrm{p}=0,001)$. As mulheres apresentaram média $15,0 \pm 5,7 \mathrm{Kgf}$, variando de $11,2 \mathrm{Kgf}$ a $18,8 \mathrm{Kgf}$; enquanto os homens atingiram média de $33,7 \pm 11,8 \mathrm{Kgf}$, variando de $26,2 \mathrm{Kgf}$ a $41,2 \mathrm{Kgf}$. O mesmo pode ser observado em relação aos valores da FAM da mão não dominante, que também variou significativamente entre os gêneros $(\mathrm{p}=0,001)$, apresentando no sexo masculino média de $30,6 \pm 11,0 \mathrm{Kgf}$, variando de $23,6 \mathrm{Kgf}$ a $37,6 \mathrm{Kgf}$; e no sexo feminino, média de $14,3 \pm 5,7 \mathrm{Kgf}$, variando de $10,4 \mathrm{Kgf}$ a $18,1 \mathrm{Kgf}$.

Em comparação da FAM com a idade, houve uma correlação negativa moderada, ou seja, quanto mais avançada a idade dos indivíduos, menor foi a FAM na mão dominante $(\mathrm{r}=-0,40)$ e também a da não dominante $(\mathrm{r}=-0,44)$; porém, quando comparamos a FAM dominante e não dominante entre idosos e não idosos, essa diferença não foi estatisticamente significativa. Não foi encontrada relação estatisticamente significante entre a DM e o IMC com a FAM dominante nem com a não dominante.

A FAM dominante variou significativamente $(\mathrm{P}=0,006)$ conforme o estado nutricional. A mão não dominante também teve sua força associada ao estado nutricional segundo ASG ( $\mathrm{P}=0,002)$, conforme os dados apresentados na Tabela 2 .
Houve correlação significativa $(\mathrm{p}<0,001)$, moderada e negativa $(r=-0,65)$ entre a FAM da mão dominante com a pontuação da ASG-PPP, ou seja, quanto maior a pontuaçáo obtida menor é a força do aperto de mão. No caso da mão não dominante, houve correlação negativa $(\mathrm{r}=-0,73)$ entre as variáveis $(\mathrm{p}<0,001)$, mostrando que o aumento da pontuação leva ao declínio da força na mão dominante. Também houve associação dos escores numéricos de recomendação de intervenção da ASG-PPP com a FAM da mão dominante $(\mathrm{p}=0,003)$ e náo dominante $(\mathrm{p}<0,001)$, conforme a Tabela 2 . Com relação à FAM e ao tipo de tumor, não houve associação estatística em nenhuma das mãos; assim como o IMC por tipo de tumor. Já a ASG-PPP associou-se com a localização tumoral $(\mathrm{p}=0,004)$, havendo maior prevalência de desnutrição moderada em pacientes com tumores de estômago, seguido por cólon e reto. Já a desnutrição grave foi mais prevalente nos pacientes com tumores de esôfago.

Não houve associação estatística da FAM, em nenhuma das mãos, com complicaçôes pós-operatórias e nem com o tempo de permanência hospitalar nessa amostra. Quando associado o tempo de internação e ASG-PPP, essa correlação também não foi encontrada.

\section{DISCUSSÃO}

O presente estudo buscou avaliar a associação entre a dinamometria manual, estado nutricional e complicaçôes pós-operatórias em pacientes cirúrgicos oncológicos; porém essa associação não foi significativa. Uma das possíveis explicaçôes seria o pequeno tamanho amostral e o pequeno número de complicaçôes, sendo estes considerados como limitaçóes do estudo.

Tabela 2. Valores da FAM de acordo com o estado nutricional segundo ASG-PPP. Pelotas, 2013

\begin{tabular}{|c|c|c|c|c|}
\hline \multirow[b]{2}{*}{ ASG-PPP } & \multicolumn{4}{|c|}{ FAM (Média \pm DP) } \\
\hline & Mão Dominante & $\mathbf{p}^{*}$ & $\begin{array}{c}\text { Mão não } \\
\text { Dominante }\end{array}$ & $\mathbf{p}^{*}$ \\
\hline \multicolumn{5}{|c|}{ Estado nutricional } \\
\hline A & $38,5(12,5) \mathrm{Kgf}$ & 0,006 & $36,2(10,5) \mathrm{Kgf}$ & 0,002 \\
\hline B & $20,5(10,4) \mathrm{Kgf}$ & & $18,6(8,9) \mathrm{Kgf}$ & \\
\hline C & $17,2(7,1) \mathrm{Kgf}$ & & $15,4(8,1) \mathrm{Kgf}$ & \\
\hline \multicolumn{5}{|l|}{ Escore numérico } \\
\hline $0-1$ & $45,8(10,8) \mathrm{Kgf}$ & 0,003 & $42,9(8,0) \mathrm{Kgf}$ & $<0,001$ \\
\hline $2-3$ & $29,0(13,4) \mathrm{Kgf}$ & & $27,1(11,1) \mathrm{Kgf}$ & \\
\hline $4-8$ & $30,7(9,4) \mathrm{Kgf}$ & & $29(4,2) \mathrm{Kgf}$ & \\
\hline$\geq 9$ & $18,9(9,3) \mathrm{Kgf}$ & & $17,1(8,3) \mathrm{Kgf}$ & \\
\hline
\end{tabular}

* Teste ANOVA

Fonte: Gonzalez MC, Borges LR, Silveira DH, Assunção MCF, Orlandi SP. Validaçáo da versão em português da Avaliaçáo Subjetiva Global Produzida pelo Paciente. Revista brasileira de nutrição clínica 2010; 25 (2): 102-8. 
A FAM apresentou diferenças significativas em relação a gênero e idade. Os homens apresentaram maiores resultados, havendo um decréscimo progressivo com o avanço da idade. Estudo de Budziarecket et al. ${ }^{17}$, que avaliou a influência das variáveis como idade e gênero em indivíduos sadios, evidencia os resultados esperados, confirmando que a idade e o gênero influenciam no status da força muscular. Vale ressaltar que, até o presente momento, não há descrito na literatura valores de referência para a DM em pacientes oncológicos.

Quando avaliado o estado nutricional segundo o IMC, houve prevalência de sobrepeso e obesidade. Sabe-se que, isoladamente, o IMC não é um instrumento sensível para a avaliação de pequenas modificaçôes do estado nutricional, uma vez que pode mascarar a perda de massa corporal magra ${ }^{17}$. Já a perda de peso tem maior impacto sobre a FAM do que o IMC, demonstrando a superioridade e especificidade da ASG-PPP como método padrão-ouro em pacientes oncológicos. Além de detectar precocemente as alteraçóes nutricionais, a ASG-PPP também é considerada um instrumento para identificar pacientes com maior risco de mortalidade ${ }^{18}$. A ASG-PPP, neste estudo, revelou que $74 \%$ dos pacientes estavam desnutridos ou em risco de desnutrição. Em um estudo comparativo com outra ferramenta de avaliação nutricional, Bauer et al. ${ }^{10}$ também encontraram uma prevalência de $74 \%$ de desnutrição em pacientes com câncer. Duval et al. ${ }^{19}$, em estudo realizado na cidade de Pelotas com pacientes do Programa de Internaçáo Domiciliar Interdisciplinar, relataram prevalência de 80\% de desnutrição ou risco de desnutrição.

Em relação aos valores da FAM e às categorias do estado nutricional dos pacientes segundo ASG-PPP, observou-se que a FAM reduziu significativamente na presença de desnutrição. $\mathrm{O}$ mesmo pode ser observado no estudo de Flood et al. ${ }^{20}$ com 217 pacientes com câncer avaliados em um hospital da Austrália. Esses resultados confirmam a sensibilidade existente entre o estado de desnutrição e a perda de músculo esquelético, evidenciando o declínio resultante da força muscular e sua funcionalidade no paciente oncológico. $\mathrm{O}$ agravo do quadro da desnutrição, juntamente com a perda de massa magra e de força, é frequente em pacientes oncológicos, e interfere na funcionalidade e na qualidade de vida ${ }^{11}$.

Em relação ao tempo de internação hospitalar, Burdenet al. ${ }^{21}$, em estudo com pacientes pré-operatórios com câncer colorretal, obtiveram média de 26,6 dias, sendo que os pacientes que tiveram uma perda de peso $>10 \%$ resultaram com mais longa permanência hospitalar. Já no estudo de Humphreys et al. ${ }^{8}$, que avaliou a medida da FAM de 50 pacientes oncológicos em internação hospitalar, apesar do menor tempo de internação encontrado (média de 10 dias), houve associaçáo do tempo de permanência com a dinamometria manual, demonstrando entáo que a função muscular diminuiu conforme o maior tempo de permanência hospitalar.

Não foi encontrada associação da medida da FAM com a ocorrência de mortalidade no presente trabalho. Norman et al. ${ }^{9}$ destacam em seu estudo que a FAM reduzida mostrou forte correlação com complicaçôes pós-operatórias e morte no período de um ano, elevando o potencial preditivo da dinamometria sobre a morbidade e mortalidade a curto e longo prazos.

\section{CONCLUSÃO}

Neste estudo, não houve associação da DM com complicaçôes pós-operatórias, bem como não esteve associada com o tempo de internação nem com mortalidade em pacientes submetidos à cirurgia oncológica; porém, quando comparada com a ferramenta considerada padrão-ouro em indivíduos oncológicos (ASG-PPP), esta se mostrou sensível e com boa correlação para a avaliação do estado nutricional.

Portanto, deve-se considerar a necessidade da realização de futuros estudos utilizando a DM como ferramenta de avaliação nutricional em amostras com diferentes tipos de cânceres, estadiamento e com maior tamanho amostral de pacientes oncológicos, a fim de proporcionar um melhor manejo e intervenção nutricional no paciente oncológico cirúrgico.

\section{CONTRIBUIÇÕES}

Vanessa Regina Limberger participou da concepção e redação do artigo, coleta de dados, análises e interpretação dos resultados. Carla Alberici Pastore e Renata Torres Abib participaram da concepçáo do artigo, análises, interpretação dos dados e revisão do artigo.

Declaraçáo de Conflito de Interesses: Nada a Declarar.

\section{REFERÊNCIAS}

1. World Health Organization. World Cancer Report, 2008. International Agency for Research on Cancer. Lyon: IARC, 2009.

2. Instituto Nacional do Câncer José Alencar da Silva. Estimativa 2014 - Incidência de Câncer no Brasil. Rio de Janeiro: INCA, 2014.

3. Instituto Nacional de Câncer José Alencar da Silva. Consenso Nacional de Nutrição Oncológica. Rio de Janeiro: INCA, 2011. v.II.

4. Correa ML, Campos AC. Prevalence of hospital malnutrition in LatinAmerica: themulticenter ELAN study. Nutrition 2003; 19(10):823-25. 
5. Barbosa-Silva MCG, Barros AJD. Avaliação Nutricional Subjetiva. Parte 1 - revisão de sua validade após duas décadas de uso. Arq Gastroenterol 2002; 39(3):181-7.

6. Muscaritoli M, Anker SD, Argilés J, Aversa Z, Bauer JM, Biolo G, et al. Consensus definition of sarcopenia, cachexia and pre-cachexia: joint document elaborated by Special Interest Groups (SIG) "cachexia-anorexia in chronic wasting diseases" and "nutrition in geriatrics". Clinical nutrition 2010; 29(2): 154-9.

7. Fearon K, Strasser F, Anker SD, Bosaeus I, Bruera E, Fainsinger RL, et al. Definition and classification of cancer cachexia: an international consensus. Lancet Oncol 2011; 12(5): 489-95.

8. Humphreys J, Maza P, Hirsch S, Barrera G, Gattas V, Bunout D. Muscle strength as a predictor of loss of functional status in hospitalized patients. Nutrition 2002; 18: 616-20.

9. Norman K, Stobäus N, Gonzalez MC, Schulzke JD, Pirlich M. Hand grip strength: Outcome predictor and marker of nutritional status. Clinical nutrition 2011; 30: 135-42.

10. Bauer J, Capra S, Ferguson M. Use of the scored PatientGenerated Subjective Global Assessment (PG-SGA) as a nutrition assessment tool in patients with cancer. Eur J Clin Nutr 2002; 58: 779-85.

11. Muscaritoli M, Bossola M, Aversa Z, Bellantone R, Rossi Fanelli F. Prevention and treatment of cancer cachexia: new insights into an old problem. Eur J Cancer 2006; 42(1): 31-41.

12. Associação Brasileira de Empresas de Pesquisa (ABEP). 2013. Disponível em: <http://www.abep.org.br>.
13. World Health Organization. Physical status: the use and interpretation of antropometry. WHO - Technical Report series (854), 1995.

14. Lipschitz, DA. Screening for nutritional status in the elderly. Primary care 1994; 21(1): 55-67.

15. Gonzalez MC, Borges LR, Silveira DH, Assunção MCF, Orlandi SP. Validação da versão em português da avaliação subjetiva global produzida pelo paciente. Revista brasileira de nutrição clínica 2010; 25(2): 102-8.

16. Schlüssel MM, Anjos LA, Kac G. A dinamometria manual e seu uso na avaliação nutricional. Revista de nutrição 2008; 21(2): 223-35.

17. Budziareck MB, Duarte RRP, Barbosa-Silva MCG. Reference values and determinants for handgrip strenght in healthy subjects. Clinical nutrition 2008; 27: 357-62.

18. Colling C, Duval PA, Silveira DH. Pacientes submetidos à quimioterapia: avaliação nutricional prévia. Revista brasileira de cancerologia 2012; 58(4): 611-7.

19. Duval P, Vargas BL, Fripp JC, Arrieira IC, Lazzeri B, Destri K et al. Caquexia em Pacientes Oncológicos Internados em um Programa de Internação Domiciliar Interdisciplinar. Revista brasileira de cancerologia 2010; 56(2): 207-12.

20. Flood A, Chung A, Parker H, Kearns V, O’Sullivan TA. The use of hand grip strength as a predictor of nutrition status in hospital patients. Clinical nutrition 2014; 33(1): 106-14.

21. Burden ST, Hill J, Shaffer JL, Todd C. Nutritional status of preoperative colorectal cancer patients. J Hum Nutr Diet 2010; 23: 402-7. 


\section{Abstract}

Introduction: The manual dynamometer is a method of nutritional assessment and prognostic marker in pre-surgical oncological patients. Objectives: To assess the association between the manual dynamometer, sociodemographic and clinical profile and outcome of hospitalization on oncological patients undergoing surgery in a public hospital in the south of Brazil. Method: Longitudinal observation study made at the Hospital of the Federal University of Pelotas with cancer patients which have undertaken their first oncological procedure along the months of May to November 2013. The patients' nutritional status were evaluated through the Body Mass Index and the Patient Generated Subjective Global Assessment, the handgrip strength was measured through a manual dynamometer and the complications were identified on the patients' medical records along the time they have been hospitalized. Results: 23 patients were assessed, being $52,2 \%$ male and $47,8 \%$ of sample with anal and cervical cancer. It was found an association between the handgrip strength and the nutritional status according Patient Generated Subjective Global Assessment categories: well-nourished risk of malnutrition, severe malnutrition, and score obtained as well as the Patient Generated Subjective Global Assessment was associated to the tumor location. The post-operative issues occurred on 21,7\% patients, although with no statistical association to their handgrip strength. Conclusion: The manual dynamometer have association to the nutritional status based on the Patient Generated Subjective Global Assessment, decreasing according it was grown worse, however it was not associated with post-operative issues, hospitalization period and mortality in the researched sample.

Key words: Muscle Strength Dynamometer; Hand Strength; Nutrition Assessment, Inpatients; Neoplasms; Observational Study

\section{Resumen}

Introducción: La dinamometría de fuerza muscular es un método de evaluación nutricional y un marcador de pronóstico en pacientes con cáncer pre-quirúrgicos. Objetivos: Verificar se hay asociación entre la dinamometría manual, el perfil sociodemográfico y clínico, y el resultado de la hospitalización de los pacientes con cáncer sometidos a cirugía en hospital público en el sur de Brasil. Método: Estudio longitudinal observacional realizado en el Hospital Escuela de la Universidad Federal de Pelotas, entre mayo y noviembre de 2013, en pacientes afectados de cáncer que se sometieron a la primera cirugía oncológica. El estado nutricional fue medido a través del Índice de Masa Corporal y de la Evaluación Subjetiva Global Generada por el paciente, la fuerza de apretón de manos fue medida a través de la dinamometría y las complicaciones fueron identificadas en los registros de los pacientes durante la hospitalización. Resultados: Fueron avaluados 23 pacientes, el 52,2\% fueron varones, delos cuales el 47,8\% de la muestra presentando cáncer de colon y recto. Hubo asociación entre la fuerza del apretón de manos y la situación nutricional según la Evaluación Subjetiva Global Generada por el paciente de acuerdo a las categorías: bien nutrido, riesgo de desnutrición y desnutrición aguda y la puntuación obtenida, así como se asoció con la ubicación del tumor. Complicaciones postoperatorias ocurrieron en el $21,7 \%$ de los pacientes, sin embargo sin ningún vínculo estadístico con la fuerza de apretón de manos. Conclusión: La dinamometría manual se asoció con la situación nutricional mediante Evaluación Subjetiva Global Generada por el paciente, disminuyendo conforme su agravamiento, pero no estuvo asociada con complicaciones postoperatorias, ni con el tiempo de hospitalización o la mortalidad en esta muestra.

Palabras clave: Dinamómetro de Fuerza Muscular; Fuerza de la Mano; Evaluación Nutricional, Pacientes Internos; Neoplasias; Estudio Observacional 